\title{
A Bijective Proof of Borchardt's Identity
}

\author{
Dan Singer \\ Minnesota State University, Mankato \\ dan. singer@mnsu.edu
}

Submitted: Jul 28, 2003; Accepted: Jul 5, 2004; Published: Jul 26, 2004

\begin{abstract}
We prove Borchardt's identity

$$
\operatorname{det}\left(\frac{1}{x_{i}-y_{j}}\right) \operatorname{per}\left(\frac{1}{x_{i}-y_{j}}\right)=\operatorname{det}\left(\frac{1}{\left(x_{i}-y_{j}\right)^{2}}\right)
$$
\end{abstract}

by means of sign-reversing involutions.

Keywords: Borchardt's identity, determinant, permanent, sign-reversing involution, alternating sign matrix.

MR Subject Code: 05A99

\section{Introduction}

In this paper we present a bijective proof of Borchardt's identity, one which relies only on rearranging terms in a sum by means of sign-reversing involutions. The proof reveals interesting properties of pairs of permutations. We will first give a brief history of this identity, indicating methods of proof.

The permanent of a square matrix is the sum of its diagonal products:

$$
\operatorname{per}\left(a_{i j}\right)_{i, j=1}^{n}=\sum_{\sigma \in S_{n}} \prod_{i=1}^{n} a_{i \sigma(i)},
$$

where $S_{n}$ denotes the symmetric group on $n$ letters. In 1855, Borchardt proved the following identity, which expresses the product of the determinant and the permanent of a certain matrix as a determinant [1]:

\section{Theorem 1.1.}

$$
\operatorname{det}\left(\frac{1}{x_{i}-y_{j}}\right) \operatorname{per}\left(\frac{1}{x_{i}-y_{j}}\right)=\operatorname{det}\left(\frac{1}{\left(x_{i}-y_{j}\right)^{2}}\right)
$$


Borchardt proved this identity algebraically, using Lagrange's interpolation formula. In 1859, Cayley proved a generalization of this formula for $3 \times 3$ matrices [4]:

Theorem 1.2. Let $A=\left(a_{i j}\right)$ be a $3 \times 3$ matrix with non-zero entries, and let $B$ and $C$ be $3 \times 3$ matrices whose $(i, j)$ entries are $a_{i j}^{2}$ and $a_{i j}^{-1}$, respectively. Then

$$
\operatorname{det}(A) \operatorname{per}(A)=\operatorname{det}(B)+2\left(\prod_{i, j} a_{i j}\right) \operatorname{det}(C) \text {. }
$$

When the matrix $A$ in this identity is equal to $\left(\left(x_{i}-y_{j}\right)^{-1}\right)$, the matrix $C$ is of rank no greater than 2 and has determinant equal to zero. Cayley's proof involved rearranging the terms of the product $\operatorname{det}(A) \operatorname{per}(A)$. In 1920, Muir gave a general formula for the product of a determinant and a permanent [8]:

Theorem 1.3. Let $P$ and $Q$ be $n \times n$ matrices. Then

$$
\operatorname{det}(P) \operatorname{per}(Q)=\sum_{\sigma \in S_{n}} \epsilon(\sigma) \operatorname{det}\left(P_{\sigma} * Q\right)
$$

where $P_{\sigma}$ is the matrix whose $i^{\text {th }}$ row is the $\sigma(i)^{\text {th }}$ row of $P, P_{\sigma} * Q$ is the Hadamard product, and $\epsilon(\sigma)$ denotes the sign of $\sigma$.

Muir's proof also involved a simple rearranging of terms. In 1960, Carlitz and Levine generalized Cayley's identity as follows [3]:

Theorem 1.4. Let $A=\left(a_{i j}\right)$ be an $n \times n$ matrix with non-zero entries and rank $\leq 2$. Let $B$ and $C$ be $n \times n$ matrices whose $(i, j)$ entries are $a_{i j}^{-1}$ and $a_{i j}^{-2}$, respectively. Then

$$
\operatorname{det}(B) \operatorname{per}(B)=\operatorname{det}(C) \text {. }
$$

Carlitz and Levine proved this theorem by setting $P=Q=B$ in Muir's identity and showing, by means of the hypothesis regarding the rank of $A$, that each of the terms $\operatorname{det}\left(B_{\sigma} * B\right)$ is equal to zero for permutations $\sigma$ not equal to the identity.

As Bressoud observed in [2], Borchardt's identity can be proved by setting $a=1$ in the Izergin-Korepin formula [5][6] quoted in Theorem 1.5 below. This determinant evaluation, expressed as a sum of weights of $n \times n$ alternating sign matrices, formed the basis of Kuperberg's proof of the alternating sign matrix conjecture [7] and Zeilberger's proof of the refined conjecture [9].

Theorem 1.5. Let $\mathcal{A}_{n}$ denote the set of $n \times n$ alternating sign matrices. Given $A=$ $\left(a_{i j}\right) \in \mathcal{A}_{n}$, let $(i, j)$ be the vertex in row $i$, column $j$ of the corresponding six-vertex model, let $N(A)=\operatorname{card}\left\{(i, j) \in[n] \times[n]: a_{i j}=-1\right\}$, let $\mathcal{I}(A)=\sum_{i<k} \sum_{j>l} a_{i j} a_{k l}$, and let $H(A), V(A), S E(A), S W(A), N E(A), N W(A)$ be, respectively, the sets of horizontal, 
vertical, southeast, southwest, northeast, and northwest vertices of the six-vertex model of A. Then for indeterminants $a, x_{1}, \ldots, x_{n}$ and $y_{1}, \ldots, y_{n}$ we have

$$
\begin{gathered}
\operatorname{det}\left(\frac{1}{\left(x_{i}+y_{j}\right)\left(a x_{i}+y_{j}\right)}\right) \frac{\prod_{i, j=1}^{n}\left(x_{i}+y_{j}\right)\left(a x_{i}+y_{j}\right)}{\prod_{1 \leq i<j \leq n}\left(x_{i}-x_{j}\right)\left(y_{i}-y_{j}\right)}= \\
\sum_{A \in \mathcal{A}_{n}}(-1)^{N(A)}(1-a)^{2 N(A)} a^{\frac{1}{2} n(n-1)-\mathcal{I}(A)} \times \\
\prod_{(i, j) \in V(A)} x_{i} y_{j} \prod_{(i, j) \in N E(A) \cup S W(A)}\left(a x_{i}+y_{j}\right) \prod_{(i, j) \in N W(A) \cup S E(A)}\left(x_{i}+y_{j}\right) .
\end{gathered}
$$

This paper is organized as follows. In Section 2 we describe a simple combinatorial model of Borchardt's identity, and in Section 3 we prove the identity by means of signreversing involutions.

\section{Combinatorial Model of Borchardt's Identity}

Borchardt's identity can be boiled down to the following statement:

Lemma 2.1. Borchardt's identity is true if and only if, for all fixed vectors of non-negative integers $p, q \in \mathbf{N}^{n}$,

$$
\begin{aligned}
& \sum \sum_{(a, b) \in N^{n} \times N^{n}} \epsilon(\sigma)=0 \text {, } \\
& (\sigma, \tau) \in S_{n} \times S_{n} \quad(a, b) \in \mathbf{N}^{n} \times \mathbf{N}^{n} \\
& \sigma \neq \tau \quad a+b=p \\
& a \circ \sigma^{-1}+b \circ \tau^{-1}=q
\end{aligned}
$$

where $x \circ \alpha$ is the vector whose $i^{\text {th }}$ entry is $x_{\alpha(i)}$.

Proof. Borchardt's identity may be regarded as a polynomial identity in the commuting variables $x_{i}$ and $y_{i}, 1 \leq i \leq n$. It is equivalent to

$$
\operatorname{det}\left(\left(1-\frac{y_{j}}{x_{i}}\right)^{-1}\right) \operatorname{per}\left(\left(1-\frac{y_{j}}{x_{i}}\right)^{-1}\right)=\operatorname{det}\left(\left(1-\frac{y_{j}}{x_{i}}\right)^{-2}\right)
$$

which is a statement about formal power series. Setting $a_{i j}=\left(1-\frac{y_{j}}{x_{i}}\right)^{-1}$, this is equivalent to

$$
\sum_{(\sigma, \tau) \in S_{n} \times S_{n}} \epsilon(\sigma) \prod_{i=1}^{n} a_{i \sigma(i)} a_{i \tau(i)}=\sum_{\sigma \in S_{n}} \epsilon(\sigma) \prod_{i=1}^{n} a_{i \sigma(i)}^{2} .
$$


This in turn is equivalent to

$$
\sum_{\substack{(\sigma, \tau) \in S_{n} \times S_{n} \\ \sigma \neq \tau}} \epsilon(\sigma) \prod_{i=1}^{n} a_{i \sigma(i)} a_{i \tau(i)}=0
$$

If we expand each entry $a_{i j}$ as a formal power series and write

$$
a_{i j}=\sum_{p \geq 0} \frac{y_{j}^{p}}{x_{i}^{p}}
$$

then equation (2.2) becomes

$$
\sum_{\substack{(\sigma, \tau) \in S_{n} \times S_{n} \\ \sigma \neq \tau}} \epsilon(\sigma) \sum_{(a, b) \in \mathbf{N}^{n} \times \mathbf{N}^{n}} \prod_{i=1}^{n}\left(\frac{y_{\sigma(i)}}{x_{i}}\right)^{a_{i}}\left(\frac{y_{\tau(i)}}{x_{i}}\right)^{b_{i}}=0 .
$$

Collecting powers of $x_{i}$ and $y_{i}$ and extracting the coefficient of $\prod_{i=1}^{n} \frac{y_{i}^{q_{i}}}{x_{i}^{p_{i}}}$ for each $(p, q) \in$ $\mathbf{N}^{n} \times \mathbf{N}^{n}$, we obtain equation (2.1).

We can now use equation (2.1) as the basis for a combinatorial model of Borchardt's identity. For each ordered pair of vectors $(p, q) \in \mathbf{N}^{n} \times \mathbf{N}^{n}$ we define the set of configurations $C(p, q)$ by

$$
C(p, q)=\left\{\begin{array}{c}
(\sigma, \tau, a, b) \in S_{n} \times S_{n} \times \mathbf{N}^{n} \times \mathbf{N}^{n}: \\
\sigma \neq \tau, a+b=p, a \circ \sigma^{-1}+b \circ \tau^{-1}=q
\end{array}\right\} .
$$

The weight of a configuration $(\sigma, \tau, a, b)$ is defined to be

$$
w(\sigma, \tau, a, b)=\epsilon(\sigma)
$$

By Lemma 2.1, Borchardt's identity is equivalent to the statement that

$$
\sum_{z \in C(p, q)} w(z)=0
$$

We will prove this identity by means of sign-reversing involutions, which pair off configurations having opposite weights. 


\section{Proof of Borchardt's Identity}

The properties of the configuration $(\sigma, \tau, a, b) \in C(p, q)$ can be conveniently summarized by the following diagram: imagine an $n \times n$ board with certain of its cells labelled by red numbers and blue numbers. A cell may have no label, a red label, a blue label, or one of each. At least one cell must have only one label. There is exactly one red label and exactly one blue label in each row and in each column. The red label in row $i$ and column $\sigma(i)$ is $a_{i}$, and the blue label in row $i$ and column $\tau(i)$ is $b_{i}$. The $i^{t h}$ row sum is equal to $p_{i}$ and the $i^{t h}$ column sum is equal to $q_{i}$. The weight of the board is equal to $\epsilon(\sigma)$, the sign of $\sigma$. An illustration of the board $B_{1}$ corresponding to the configuration

$$
\left((1)(2)(3)(4),(1)(234),\left(a_{1}, a_{2}, a_{3}, a_{4}\right),\left(b_{1}, b_{2}, b_{3}, b_{4}\right)\right)
$$

is contained in Figure 3.1 below. $C(p, q)$ can be identified with the totality of such boards.

Figure 3.1: $B_{1}$

\begin{tabular}{|l|l|l|l|}
\hline$a_{1}$ & & & \\
\hline$b_{1}$ & & & \\
\hline & $a_{2}$ & $b_{2}$ & \\
& & & \\
\hline & & $a_{3}$ & $b_{3}$ \\
& & & \\
& & & \\
\hline & & & \\
\hline
\end{tabular}

If $\theta$ is a sign-reversing involution of $C(p, q)$, then it must satisfy

$$
\theta(\sigma, \tau, a, b)=\left(\sigma^{\prime}, \tau^{\prime}, a^{\prime}, b^{\prime}\right),
$$

where $\epsilon\left(\sigma^{\prime}\right)=-\epsilon(\sigma)$. One way to produce $\sigma^{\prime}$ is to transpose two of the rows or two of the columns in the corresponding diagram. One must be careful, however, to preserve row and column sums. If two of the row sums are the same, or if two of the column sums are the same, there is no problem. We prove this formally in the next lemma. 
Lemma 3.1. If $p$ or $q$ has repeated entries then equation (2.3) is true.

Proof. Let $\alpha$ represent the transposition which exchanges the indices $i$ and $j$. If $p_{i}=p_{j}$ then

$$
(\sigma, \tau, a, b) \mapsto(\sigma \alpha, \tau \alpha, a \circ \alpha, b \circ \alpha)
$$

is a sign-reversing involution of $C(p, q)$. If $q_{i}=q_{j}$ then

$$
(\sigma, \tau, a, b) \mapsto(\alpha \sigma, \alpha \tau, a, b)
$$

is a sign-reversing involution of $C(p, q)$.

We will henceforth deal with configuration sets $C(p, q)$ in which neither $p$ nor $q$ has repeated entries. We will describe two other classes of board rearrangements both geometrically and algebraically, then prove that they can be combined to show that equation (2.3) is true.

The first class of rearrangements we will call $\phi$. Let $(\sigma, \tau, a, b) \in C(p, q)$ be given. Let $i$ be any index such that $a_{i} \geq a_{\gamma(i)}$ and $b_{i} \geq b_{\gamma^{-1}(i)}$, where $\gamma=\sigma^{-1} \tau$ and $\sigma(i) \neq \tau(i)$. Then $a_{i}$ and $b_{i}$ are both in row $i, a_{\gamma(i)}$ is in the same column as $b_{i}$, and $b_{\gamma^{-1}(i)}$ is in the same column as $a_{i}$. To produce the rearrangement $\phi_{i}(\sigma, \tau, a, b)=\left(\sigma^{\prime}, \tau^{\prime}, a^{\prime}, b^{\prime}\right)$, we will first replace the red label $a_{i}$ by the red label $b_{i}-b_{\gamma^{-1}(i)}+a_{\gamma(i)}$, replace the blue label $b_{i}$ by the blue label $a_{i}-a_{\gamma(i)}+b_{\gamma^{-1}(i)}$, then switch the columns $\sigma(i)$ and $\tau(i)$. For the example, the $\phi_{2}$-rearrangement of the board $B_{1}$ in Figure 3.1 is the board $B_{2}$ depicted in Figure 3.2 below. It is easy to verify that row and column sums are preserved and that the sign of the original board has been reversed. The algebraic definition of $\phi_{i}(\sigma, \tau, a, b)$ is $\left(\sigma^{\prime}, \tau^{\prime}, a^{\prime}, b^{\prime}\right)$, where

$$
\begin{gathered}
\sigma^{\prime}=(\sigma(i) \tau(i)) \sigma, \\
\tau^{\prime}=(\sigma(i) \tau(i)) \tau, \\
a_{j}^{\prime}= \begin{cases}a_{j} & \text { if } j \neq i \\
b_{i}-b_{\gamma^{-1}(i)}+a_{\gamma(i)} & \text { if } j=i\end{cases}
\end{gathered}
$$

and

$$
b_{j}^{\prime}= \begin{cases}b_{j} & \text { if } j \neq i \\ a_{i}-a_{\gamma(i)}+b_{\gamma^{-1}(i)} & \text { if } j=i\end{cases}
$$

The second class of rearrangements we will call $\psi$. Let $(\sigma, \tau, a, b) \in C(p, q)$ be given. Let $i$ be any index such that $a_{\sigma^{-1}(i)} \geq a_{\tau^{-1}(i)}$ and $b_{\tau^{-1}(i)} \geq b_{\sigma^{-1}(i)}$, where $\sigma^{-1}(i) \neq \tau^{-1}(i)$. Then $a_{\sigma^{-1}(i)}$ and $b_{\tau^{-1}(i)}$ are both in column $i, b_{\sigma^{-1}(i)}$ is in the same row as $a_{\sigma^{-1}(i)}$, and $a_{\tau^{-1}(i)}$ is in the same column as $b_{\tau^{-1}(i)}$. To produce the rearrangement $\psi_{i}(\sigma, \tau, a, b)=\left(\sigma^{\prime}, \tau^{\prime}, a^{\prime}, b^{\prime}\right)$, we will first replace the red label $a_{\sigma^{-1}(i)}$ by the red label $b_{\tau^{-1}(i)}-b_{\sigma^{-1}(i)}+a_{\tau^{-1}(i)}$, replace 
Figure 3.2: $B_{2}=\phi_{2}\left(B_{1}\right)$

\begin{tabular}{|c|c|c|c|}
\hline$a_{1}$ & & & \\
$b_{1}$ & & & \\
\hline & $a_{2}-a_{3}+b_{4}$ & $b_{2}-b_{4}+a_{3}$ & \\
\hline & $a_{3}$ & & $b_{3}$ \\
\hline & & & \\
\hline & & $b_{4}$ & $a_{4}$ \\
\hline
\end{tabular}

the blue label $b_{\tau^{-1}(i)}$ by the blue label $a_{\sigma^{-1}(i)}-a_{\tau^{-1}(i)}+b_{\sigma^{-1}(i)}$, then switch the rows $\sigma^{-1}(i)$ and $\tau^{-1}(i)$. For example, the $\psi_{2}$-rearrangement of the board $B_{1}$ in Figure 3.1 is the board $B_{3}$ depicted in Figure 3.3 below. The rearrangements $\psi$ are related to the rearrangements $\phi$ in the sense that if we start with a board, reverse the rows of row and column, apply $\phi_{i}$, then reverse the roles of row and column again, then we obtain $\psi_{i}$. Hence row and column sums are preserved and the sign of the original board is reversed. The algebraic definition of $\psi_{i}(\sigma, \tau, a, b)$ is $\left(\sigma^{\prime}, \tau^{\prime}, a^{\prime}, b^{\prime}\right)$, where

$$
\begin{aligned}
& \sigma^{\prime}=\sigma\left(\sigma^{-1}(i) \tau^{-1}(i)\right), \\
& \tau^{\prime}=\tau\left(\sigma^{-1}(i) \tau^{-1}(i)\right), \\
& a_{j}^{\prime}= \begin{cases}a_{j} & \text { if } j \notin\left\{\sigma^{-1}(i), \tau^{-1}(i)\right\} \\
a_{\tau^{-1}(i)} & \text { if } j=\sigma^{-1}(i) \\
b_{\tau^{-1}(i)}-b_{\sigma^{-1}(i)}+a_{\tau^{-1}(i)} & \text { if } j=\tau^{-1}(i)\end{cases}
\end{aligned}
$$


Figure 3.3: $B_{3}=\psi_{2}\left(B_{1}\right)$

\begin{tabular}{|l|l|l|l|}
\hline$a_{1}$ & & & \\
$b_{1}$ & & & \\
\hline & $a_{2}-a_{4}+b_{2}$ & & $a_{4}$ \\
\hline & & $a_{3}$ & $b_{3}$ \\
\hline & & & \\
\hline & $b_{4}-b_{2}+a_{4}$ & $b_{2}$ & \\
& & & \\
\hline
\end{tabular}

and

$$
b_{j}^{\prime}= \begin{cases}b_{j} & \text { if } j \notin\left\{\sigma^{-1}(i), \tau^{-1}(i)\right\} \\ b_{\sigma^{-1}(i)} & \text { if } j=\tau^{-1}(i) \\ a_{\sigma^{-1}(i)}-a_{\tau^{-1}(i)}+b_{\sigma^{-1}(i)} & \text { if } j=\sigma^{-1}(i) .\end{cases}
$$

The mappings $\phi_{i}$ and $\psi_{i}$ are not defined on all of $C(p, q)$. We will prove, however, that they are sign-reversing involutions when restricted to their domains of definition. Let $z=(\sigma, \tau, a, b) \in C(p, q)$ be given. Set $\gamma=\sigma^{-1} \tau$. We define

$$
\begin{gathered}
A(z)= \\
\left\{i \leq n: \sigma(i) \neq \tau(i) \& a_{i} \geq a_{\gamma(i)} \& b_{i} \geq b_{\gamma^{-1}(i)}\right\}
\end{gathered}
$$

and

$$
\begin{gathered}
B(z)= \\
\left\{i \leq n: \sigma^{-1}(i) \neq \tau^{-1}(i) \& a_{\sigma^{-1}(i)} \geq a_{\tau^{-1}(i)} \& b_{\tau^{-1}(i)} \geq b_{\sigma^{-1}(i)}\right\} .
\end{gathered}
$$


Then $\phi_{i}(z)$ is defined if $i \in A(z)$ and $\psi_{i}(z)$ is defined if $i \in B(z)$ for each $z \in C(p, q)$. One concern is that $A(z) \cup B(z)$ is empty for some $z$, so that neither $\phi_{i}$ nor $\psi_{i}$ can be applied for any $i$. The next lemma states that this will never happen.

Lemma 3.2. For each $z \in C(p, q), A(z) \cup B(z) \neq \emptyset$.

Proof. Let $z=(\sigma, \tau, a, b) \in C(p, q)$ be given. Set $\gamma=\sigma^{-1} \tau$. Let

$$
I=\{i \leq n: \sigma(i) \neq \tau(i)\}
$$

and

$$
J=\left\{i \leq n: \sigma^{-1}(i) \neq \tau^{-1}(i)\right\}
$$

Then we have

$$
A(z)=\left\{i \in I: a_{i} \geq a_{\gamma(i)} \& b_{i} \geq b_{\gamma^{-1}(i)}\right\}
$$

and

$$
B(z)=\left\{i \in J: a_{\sigma^{-1}(i)} \geq a_{\tau^{-1}(i)} \& b_{\tau^{-1}(i)} \geq b_{\sigma^{-1}(i)}\right\} .
$$

We will also set

$$
B^{\prime}(z)=\left\{i \in I: a_{i} \geq a_{\gamma^{-1}(i)} \& b_{\gamma^{-1}(i)} \geq b_{i}\right\} .
$$

It is easy to see that

$$
i \in B(z) \Leftrightarrow \sigma^{-1}(i) \in B^{\prime}(z) .
$$

Hence we need only show that $A(z) \cup B^{\prime}(z) \neq \emptyset$.

Suppose $A(z) \cup B^{\prime}(z)=\emptyset$. Let

$$
X=\left\{i \in I: a_{i}>a_{\gamma(i)}\right\} .
$$

We claim that $X$ must be empty. If it isn't, let $p \in X$ be given. Then $a_{p}>a_{\gamma(p)}$. Since we are assuming $A(z)=\emptyset$, we must have $b_{p}<b_{\gamma^{-1}(p)}$. Since we are also assuming $B^{\prime}(z)=\emptyset$, we must have $a_{p}<a_{\gamma^{-1}(p)}$. Set $q=\gamma^{-1}(p)$. Then $a_{q}>a_{\gamma(q)}$. Since $\gamma$ permutes the indices in $I$, we have $q \in X$. Hence $i \in X \Rightarrow \gamma^{-1}(i) \in X$ for all $i \in X$. But this implies

$$
a_{p}<a_{\gamma^{-1}(p)}<a_{\gamma^{-2}(p)}<\cdots,
$$

which is impossible because $\gamma$ is of finite order. Hence our claim that $X$ is empty is true.

Since $X$ is empty, we must have $a_{i} \leq a_{\gamma(i)}$ for all $i \in I$. This implies

$$
a_{i} \leq a_{\gamma(i)} \leq a_{\gamma^{2}(i)} \leq \cdots
$$

for all $i \in I$. Since $\gamma$ has finite order, this implies that $a_{\gamma^{k}(i)}=a_{i}$ for all integers $k$ and every index $i \in I$. In particular, $a_{i}=a_{\gamma(i)}$ for all $i \in I$. Since we are assuming $A(z)$ is empty, we must have $b_{i}<b_{\gamma^{-1}(i)}$ for all $i \in I$. Let $i_{0} \in I$ be any index in $I$, which we know to be non-empty because $\sigma \neq \tau$. Then

$$
b_{i_{0}}<b_{\gamma^{-1}\left(i_{0}\right)}<b_{\gamma^{-2}\left(i_{0}\right)}<\cdots .
$$

Since $\gamma$ is of fine order, this is impossible. Hence assuming $A(z) \cup B^{\prime}(z)=\emptyset$ leads to a contradiction. Therefore $A(z) \cup B^{\prime}(z)$ cannot be empty. This implies $A(z) \cup B(z) \neq \emptyset$. 
Given a configuration set $C(p, q)$, we will distinguish two special subsets,

$$
C_{A}(p, q)=\{z \in C(p, q): A(z) \neq \emptyset\}
$$

and

$$
C_{B}(p, q)=\{z \in C(p, q): B(z) \neq \emptyset\} .
$$

Lemma 3.2 assures us that $C_{A}(p, q) \cup C_{B}(p, q)=C(p, q)$. The two sets $C_{A}(p, q)$ and $C_{B}(p, q)$ are closely related to each other, in the following sense: Let $T$ denote the operator which sends a configuration to its tranpose. The precise definition of $T(\sigma, \tau, a, b)$ is $\left(\sigma^{-1}, \tau^{-1}, a \circ \sigma^{-1}, b \circ \tau^{-1}\right)$, but it is easier to think of $T(z)$ as the board corresponding to $z$ with the roles of row and column reversed. It is easy to verify that

$$
\begin{gathered}
z \in C_{A}(p, q) \Leftrightarrow T(z) \in C_{B}(q, p), \\
i \in A(z) \Leftrightarrow i \in B(T(z)),
\end{gathered}
$$

and

$$
\psi_{i}(z)=T \circ \phi_{i} \circ T(z)
$$

where $z=(\sigma, \tau, a, b)$.

We will define a sign-reversing involution $\theta_{A}$ on $C_{A}(p, q)$ and a sign-reversing involution $\theta_{B}$ on $C_{B}(p, q)$ for each pair of vectors $p$ and $q$ having no repeated entries. We will also show that both $\theta_{A}$ and $\theta_{B}$ map $C_{A}(p, q) \cap C_{B}(p, q)$ into itself. Hence a sign-reversing involution of $C(p, q)$ is $\theta$, defined by

$$
\theta(z)= \begin{cases}\theta_{A}(z) & \text { if } z \in C_{A}(p, q) \\ \theta_{B}(z) & \text { if } z \in C_{B}(p, q) \backslash C_{A}(p, q) .\end{cases}
$$

Let $z \in C_{A}(p, q)$. Let $i$ be the least integer in $A(z)$. Then we set

$$
\theta_{A}(z)=\phi_{i}(z)
$$

Having defined $\theta_{A}$, we set

$$
\theta_{B}=T \circ \theta_{A} \circ T
$$

The next two lemmas will be used to show that $\theta_{A}$ and $\theta_{B}$ have the desired properties.

Lemma 3.3. For each $z \in C_{A}(p, q)$ and $i \in A(z)$, we have $i \in A\left(\phi_{i}(z)\right), \phi_{i}(z) \in C_{A}(p, q)$, and $\phi_{i}\left(\phi_{i}(z)\right)=z$. 
Proof. Let $z=(\sigma, \tau, a, b) \in C(p, q)$ and $i \in A(z)$ be given. Set $\gamma=\sigma^{-1} \tau$. If we write $\phi_{i}(z)=\left(\sigma^{\prime}, \tau^{\prime}, a^{\prime}, b^{\prime}\right)$, defined as in equations (3.1) through (3.4), then by the geometric characterization given earlier it is easy to see that $\phi_{i}$ preserves row and column sums. Hence $\phi_{i}(z) \in C(p, q)$. Note that $\left(\sigma^{\prime}\right)^{-1} \tau^{\prime}=\sigma^{-1} \tau=\gamma$. Hence we also have

$$
a_{i}^{\prime} \geq a_{\gamma(i)}=a_{\gamma(i)}^{\prime}
$$

and

$$
b_{i}^{\prime} \geq b_{\gamma^{-1}(i)}=b_{\gamma^{-1}(i)}^{\prime}
$$

because $\gamma(i) \neq i$. Therefore $i \in A\left(\phi_{i}(z)\right)$ and $\phi_{i}(z) \in C_{A}(p, q)$. The geometric characterization of $\phi_{i}$ implies that $\phi_{i}\left(\phi_{i}(z)\right)=z$.

Lemma 3.4. Let $p$ and $q$ be vectors in $\mathbf{N}^{n}$ which contain no repeated entries. For each $z \in C_{A}(p, q)$, if $i$ is the smallest index in $A(z)$ then $i$ is also the smallest index in $A\left(\phi_{i}(z)\right)$.

Proof. Let $z=(\sigma, \tau, a, b) \in C_{A}(p, q)$ be given. Set $\gamma=\sigma^{-1} \tau$ and $\phi_{i}(z)=\left(\sigma^{\prime}, \tau^{\prime}, a^{\prime}, b^{\prime}\right)$. Let $i$ be the smallest index in $A(z)$. By Lemma 3.3 we can say that $i \in A\left(\phi_{i}(z)\right)$ and $\phi_{i}(z) \in C_{A}(p, q)$. Let $j$ be the smallest index in $A\left(\phi_{i}(z)\right)$. We wish to show that $j=i$. Suppose $j<i$. We know that

$$
a_{j}^{\prime} \geq a_{\gamma(j)}^{\prime}
$$

and

$$
b_{j}^{\prime} \geq b_{\gamma^{-1}(j)}^{\prime} .
$$

If $\gamma(j) \neq i$ and $\gamma^{-1}(j) \neq i$ then (3.13) and (3.14) become

$$
a_{j} \geq a_{\gamma(j)}
$$

and

$$
b_{j} \geq b_{\gamma^{-1}(j)}
$$

which contradicts the fact that $i$ is least in $A(z)$. So we must have $\gamma(j)=i$ or $\gamma^{-1}(j)=i$. We will show that if $\gamma(j)=i$ or $\gamma^{-1}(j)=i$ then $p_{i}=p_{j}$, contradicting our hypothesis that $p$ has no repeated entries.

Set $\widehat{z}=\phi_{j}\left(\phi_{i}(z)\right)$. By Lemma 3.3, $\widehat{z} \in C_{A}(p, q), j \in A(\widehat{z})$, and $\phi_{j}(\widehat{z})=\phi_{i}(z)$. Let us write $\widehat{z}=(\widehat{\sigma}, \widehat{\tau}, \widehat{a}, \widehat{b})$. Staying consistent with our notation up to this point, we write $\phi_{j}(\widehat{z})=\left(\widehat{\sigma}^{\prime}, \widehat{\tau}^{\prime}, \widehat{a}^{\prime}, \widehat{b}^{\prime}\right)$. Since $\phi_{i}(z)=\phi_{j}(\widehat{z})$, we must have

$$
\left(\sigma^{\prime}, \tau^{\prime}, a^{\prime}, b^{\prime}\right)=\left(\widehat{\sigma}^{\prime}, \widehat{\tau}^{\prime}, \widehat{a}^{\prime}, \widehat{b}^{\prime}\right) .
$$

In particular, we have

$$
\begin{aligned}
a_{i}^{\prime} & =\widehat{a}_{i}^{\prime}, \\
b_{i}^{\prime} & =\widehat{b}_{i}^{\prime},
\end{aligned}
$$




$$
a_{j}^{\prime}=\widehat{a}_{j}^{\prime}
$$

and

$$
b_{j}^{\prime}=\widehat{b}_{j}^{\prime}
$$

By definition, equations (3.17) through (3.20) are equivalent to

$$
\begin{aligned}
& b_{i}-b_{\gamma^{-1}(i)}+a_{\gamma(i)}=\widehat{a}_{i}, \\
& a_{i}-a_{\gamma(i)}+b_{\gamma^{-1}(i)}=\widehat{b}_{i}, \\
& a_{j}=\widehat{b}_{j}-\widehat{b}_{\gamma^{-1}(j)}+\widehat{a}_{\gamma(j)},
\end{aligned}
$$

and

$$
b_{j}=\widehat{a}_{j}-\widehat{a}_{\gamma(j)}+\widehat{b}_{\gamma^{-1}(j)} .
$$

Suppose $\gamma(j)=i$. Adding together equations (3.21) and (3.24) we obtain

$$
a_{\gamma(i)}+b_{i}=\widehat{a}_{j}+\widehat{b}_{\gamma^{-1}(j)},
$$

which is equivalent to $q_{\tau(i)}=q_{\sigma(j)}$. Since we are assuming that $q$ has no repeated entries, this implies $\tau(i)=\sigma(j)$, i.e. that $j=\gamma(i)$. Subtracting equation (3.23) from equation (3.21) and making the substitutions $\gamma(j)=i$ and $\gamma(i)=j$ we obtain

$$
b_{i}-b_{\gamma^{-1}(i)}=\widehat{b}_{\gamma^{-1}(j)}-\widehat{b}_{j}
$$

Since $i \in A(z)$ and $j \in A(\widehat{z})$, the left hand side of this equation is $\geq 0$ and the right hand side of this equation is $\leq 0$. Hence both sides are equal to zero, and this implies $b_{i}=b_{\gamma^{-1}(i)}=b_{j}$. Subtracting equation (3.24) from equation (3.22) and making the substitutions $\gamma(j)=i$ and $\gamma(i)=j$ we obtain

$$
a_{i}-a_{\gamma(i)}=\widehat{a}_{\gamma(j)}-\widehat{a}_{j}
$$

Since $i \in A(z)$ and $j \in A(\widehat{z})$, the left hand side of this equation is $\geq 0$ and the right hand side of this equation is $\leq 0$. Hence both sides are equal to zero, and this implies $a_{i}=a_{\gamma(i)}=a_{j}$. Putting everything together we have $p_{i}=a_{i}+b_{i}=a_{j}+b_{j}=p_{j}$, contrary to hypothesis. Therefore $\gamma(j)=i$ is not possible.

Now suppose $\gamma^{-1}(j)=i$. Adding together equations (3.22) and (3.23) we obtain

$$
a_{i}+b_{\gamma^{-1}(i)}=\widehat{a}_{\gamma(j)}+\widehat{b}_{j},
$$

which is equivalent to $q_{\sigma(i)}=q_{\tau(j)}$. Since we are assuming that $q$ has no repeated entries, this implies $\sigma(i)=\tau(j)$, i.e. that $i=\gamma(j)$. But we showed above that this case is not possible.

Therefore our original hypothesis that $j<i$ leads to a contradiction. Hence $j \geq i$. But $j$ is least in $A\left(\phi_{i}(z)\right)$ and $i \in A\left(\phi_{i}(z)\right)$, therefore $j=i$. Hence $i$ is least in $A\left(\phi_{i}(z)\right)$. 
Since each $\phi_{i}$ is sign-reversing, if we combine Lemmas 3.3 and 3.4 we obtain

Proposition 3.5. Let $p$ and $q$ be vectors in $\mathbf{N}^{n}$ having no repeated entries. Then $\theta_{A}$ is a sign-reversing involution of $C_{A}(p, q)$.

Since $\theta_{B}=T \circ \theta_{A} \circ T$ and $T$ is a sign-preserving involution and $\theta_{A}$ is a sign-reversing involution, Proposition 3.5 immediately gives us the following result:

Proposition 3.6. Let $p$ and $q$ be vectors in $\mathbf{N}^{n}$ having no repeated entries. Then $\theta_{B}$ is a sign-reversing involution of $C_{B}(p, q)$.

Our last task is to prove the following:

Proposition 3.7. Let $p$ and $q$ be vectors in $\mathbf{N}^{n}$ having no repeated entries. Then $\theta_{A}$ and $\theta_{B}$ both $\operatorname{map} C_{A}(p, q) \cap C_{B}(p, q)$ into itself.

Proof. We will prove this by contradiction. Let $p$ and $q$ be vectors in $\mathbf{N}^{n}$ having no repeated entries, and suppose there exists a configuration $z=(\sigma, \tau, a, b) \in C_{A}(p, q) \cap$ $C_{B}(p, q)$ such that $\theta_{A}(z) \notin C_{A}(p, q) \cap C_{B}(p, q)$. Since we know that $\theta_{A}(z) \in C_{A}(p, q)$, it must be true that $\theta_{A}(z) \notin C_{B}(p, q)$. Let $i=\min A(z)$. We will show that $A(z)=\{i\}$ and $B(z)=\{\sigma(i)\}$ or $B(z)=\{\tau(i)\}$.

Let $i_{0} \in A(z)$ be any index such that $\phi_{i_{0}}(z) \notin C_{A}(p, q) \cap C_{B}(p, q)$. This means that $\phi_{i_{0}}(z) \notin C_{B}(p, q)$, that is $B\left(\phi_{i_{0}}(z)\right)=\emptyset$. By definition, we have $\phi_{i_{0}}(z)=\left(\sigma^{\prime}, \tau^{\prime}, a^{\prime}, b^{\prime}\right)$, where $\left(\sigma^{\prime}, \tau^{\prime}, a^{\prime}, b^{\prime}\right)$ is defined as in equations (3.1) through (3.4), with $i$ replaced by $i_{0}$. For any index $j \notin\left\{\sigma\left(i_{0}\right), \tau\left(i_{0}\right)\right\}$ we have $a_{\left(\sigma^{\prime}\right)^{-1}(j)}^{\prime}=a_{\sigma^{-1}(j)}, a_{\left(\tau^{\prime}\right)^{-1}(j)}^{\prime}=a_{\tau^{-1}(j)}, b_{\left(\sigma^{\prime}\right)^{-1}(j)}^{\prime}=$ $b_{\sigma^{-1}(j)}$, and $b_{\left(\tau^{\prime}\right)^{-1}(j)}^{\prime}=b_{\tau^{-1}(j)}$. Since we are assuming $B\left(\sigma^{\prime}, \tau^{\prime}, a^{\prime}, b^{\prime}\right)=\emptyset$, it must be true that $j \notin B(\sigma, \tau, a, b)$. Hence we have established that $B(z) \subseteq\left\{\sigma\left(i_{0}\right), \tau\left(i_{0}\right)\right\}$.

We will next show that $B(z)$ contains only one element. It contains at least one element, because we are assuming that $z \in C_{B}(p, q)$. Since $i_{0} \in A(z)$, we know that $a_{i_{0}} \geq a_{\gamma\left(i_{0}\right)}$ and $b_{i_{0}} \geq b_{\gamma^{-1}\left(i_{0}\right)}$, where $\gamma=\sigma^{-1} \tau$. If $\sigma\left(i_{0}\right) \in B(z)$ then $a_{i_{0}} \geq a_{\gamma^{-1}\left(i_{0}\right)}$ and $b_{\gamma^{-1}\left(i_{0}\right)} \geq b_{i_{0}}$, forcing $b_{i_{0}}=b_{\gamma^{-1}\left(i_{0}\right)}$. If $\tau\left(i_{0}\right) \in B(z)$ then $a_{\gamma\left(i_{0}\right)} \geq a_{i_{0}}$ and $b_{i_{0}} \geq b_{\gamma\left(i_{0}\right)}$, forcing $a_{i_{0}}=a_{\gamma\left(i_{0}\right)}$. So if $B(z)=\left\{\sigma\left(i_{0}\right), \tau\left(i_{0}\right)\right\}$, then

$$
q_{\sigma\left(i_{0}\right)}=a_{i_{0}}+b_{\gamma^{-1}\left(i_{0}\right)}=a_{\gamma\left(i_{0}\right)}+b_{i_{0}}=q_{\tau\left(i_{0}\right)} .
$$

However, since $\sigma\left(i_{0}\right) \neq \tau\left(i_{0}\right)$, this means that $q$ has a repeated entry, contrary to hypothesis. So $B(z)=\left\{\sigma\left(i_{0}\right)\right\}$ or $B(z)=\left\{\tau\left(i_{0}\right)\right\}$.

We will now show that $A(z)$ contains only one element. Suppose the index $i_{0}$ we have been considering is different from $i$. By the logic above, $B(z)=\{\sigma(i)\}$ or $B(z)=\{\tau(i)\}$. However, we also know that $B(z)=\left\{\sigma\left(i_{0}\right)\right\}$ or $B(z)=\left\{\tau\left(i_{0}\right)\right\}$. If $i \in A(z)$ and $B(z)=$ $\{\sigma(i)\}$, then we must have $B(z)=\left\{\tau\left(i_{0}\right)\right\}$ and $\gamma^{-1}(i)=i_{0} \in A(z)$. But $i \in A(z)$ implies

$$
b_{i} \geq b_{\gamma^{-1}(i)}
$$


$\gamma^{-1}(i) \in A(z)$ implies

$$
a_{\gamma^{-1}(i)} \geq a_{i}
$$

and $\sigma(i) \in B(z)$ implies

$$
a_{i} \geq a_{\gamma^{-1}(i)} \text { and } b_{\gamma^{-1}(i)} \geq b_{i} .
$$

Inequalities (3.25) through (3.27) imply $a_{i}=a_{\gamma^{-1}(i)}$ and $b_{i}=b_{\gamma^{-1}(i)}$, which implies

$$
p_{i}=a_{i}+b_{i}=a_{\gamma^{-1}(i)}+b_{\gamma^{-1}(i)}=p_{\gamma^{-1}(i)},
$$

which contradicts our hypothesis that $p$ has no repeated entries. On the other hand, if $i \in A(z)$ and $B(z)=\{\tau(i)\}$, then we must have $B(z)=\left\{\sigma\left(i_{0}\right)\right\}$ and $\gamma(i)=i_{0} \in A(z)$. But $i \in A(z)$ implies

$$
a_{i} \geq a_{\gamma(i)}
$$

$\gamma(i) \in A(z)$ implies

$$
b_{\gamma(i)} \geq b_{i}
$$

and $\tau(i) \in B(z)$ implies

$$
a_{\gamma(i)} \geq a_{i} \text { and } b_{i} \geq b_{\gamma(i)}
$$

Inequalities (3.28) through (3.30) imply $a_{i}=a_{\gamma(i)}$ and $b_{i}=b_{\gamma(i)}$, which implies

$$
p_{i}=a_{i}+b_{i}=a_{\gamma(i)}+b_{\gamma(i)}=p_{\gamma(i)},
$$

which again contradicts our hypothesis that $p$ has no repeated entries. Hence $A(z)=\{i\}$.

We will now show that each of the cases

$$
A(z)=\{i\} \text { and } B(z)=\{\sigma(i)\}
$$

and

$$
A(z)=\{i\} \text { and } B(z)=\{\tau(i)\}
$$

is impossible, given our hypothesis that $\theta_{A}(z) \notin C_{A}(p, q) \cap C_{B}(p, q)$. We will record here that $A(z)=\{i\}$ implies

$$
a_{j}<a_{\gamma(j)} \text { or } b_{j}<b_{\gamma^{-1}(j)} \text { for each } j \neq i \text { in }\left\{\gamma^{k}(i): k \in \mathbf{Z}\right\}
$$

and $B(z)=\{\sigma(i)\}$ implies

$$
a_{j}<a_{\gamma^{-1}(j)} \text { or } b_{\gamma^{-1}(j)}<b_{j} \text { for each } j \neq i \text { in }\left\{\gamma^{k}(i): k \in \mathbf{Z}\right\} .
$$

We will write $\theta_{A}(z)=\phi_{i}(z)=\left(\sigma^{\prime}, \tau^{\prime}, a^{\prime}, b^{\prime}\right)$, consistent with the notation in equations (3.1) through (3.4).

First suppose that case (3.31) is true. Then (3.25) and (3.27) imply

$$
b_{i}=b_{\gamma^{-1}(i)}
$$


Since

$$
a_{\gamma(i)}^{\prime}=a_{\gamma(i)} \geq b_{i}-b_{\gamma^{-1}(i)}+a_{\gamma(i)}=a_{i}^{\prime}
$$

and $B\left(\phi_{i}(z)\right)=\emptyset$, we must have $b_{i}^{\prime}<b_{\gamma(i)}^{\prime}$, that is

$$
a_{i}-a_{\gamma(i)}+b_{\gamma^{-1}(i)}<b_{\gamma(i)} .
$$

Since $a_{i} \geq a_{\gamma(i)}$, this implies $b_{\gamma^{-1}(i)}<b_{\gamma(i)}$. Hence $b_{i}<b_{\gamma(i)}$. Let

$$
X=\left\{k \geq 0: b_{\gamma^{k}(i)}<b_{\gamma^{k+1}(i)}\right\} .
$$

Then we have just shown that $0 \in X$. Let $k_{0}$ be the largest integer in $X$ such that $0 \leq k \leq k_{0}$ implies $k \in X$. The largest integer $k_{0}$ must exist because the order of $\gamma$ is finite. We have

$$
b_{i}<b_{\gamma(i)}<\cdots<b_{\gamma^{k_{0}(i)}}<b_{\gamma^{k_{0}+1}(i)},
$$

hence $\gamma^{k_{0}+1}(i) \neq i$. Set $j=\gamma^{k_{0}+1}(i)$. We have $b_{j}>b_{\gamma^{-1}(j)}$, hence by (3.33) we must have $a_{j}<a_{\gamma(j)}$. By (3.35) and (3.36), it must be true that $\gamma(j) \neq i$, hence by (3.34) we also have $b_{j}<b_{\gamma(j)}$. This places $k_{0}+1$ in $X$, contradicting the definition of $k_{0}$. Therefore case (3.31) cannot be true.

The case (3.32) is also impossible. If we define $\widehat{z}$ by

$$
\widehat{z}=(\tau, \sigma, b, a) \in C_{A}(p, q) \cap C_{B}(p, q),
$$

then $\theta_{A}(z) \notin C_{A}(p, q) \cap C_{B}(p, q)$ implies $\theta_{A}(\widehat{z}) \notin C_{A}(p, q) \cap C_{B}(p, q)$. Hence case (3.32) with respect to $z$ is equivalent to case (3.31) with respect to $\widehat{z}$, and we have just shown this case to be impossible. Pictorially, $\widehat{z}$ is obtained from $z$ by swapping the colors red and blue.

Hence we have shown that $\theta_{A}$ maps $C_{A}(p, q) \cap C_{B}(p, q)$ into itself for all $p$ and $q$ having no repeated entries. Since $\theta_{B}=T \circ \theta_{A} \circ T$, and clearly $T$ maps $C_{A}(p, q) \cap C_{B}(p, q)$ into $C_{A}(q, p) \cap C_{B}(q, p), \theta_{B}$ also maps $C_{A}(p, q) \cap C_{B}(p, q)$ into itself.

Putting together Propositions 3.5 through 3.7, we have proved

Theorem 3.8. Let $p$ and $q$ be vectors in $\mathbf{N}^{n}$ having no repeated entries. Let $\theta$ be the map defined in equation (3.12). Then $\theta$ is a sign-reversing involution of $C(p, q)$.

Theorem 3.8 implies that equation (2.3) is true, hence we have a bijective proof of Borchardt's identity. 


\section{References}

[1] C. W. Borchardt, Bestimmung der symmetrischen Verbindungen vermittelst ihrer erzeugenden Funktion, Crelle's Journal 53 (1855), 193-198.

[2] D. M. Bressoud, Three alternating sign matrix identities in search of bijective proofs, Advances in Applied Mathematics 27 (2001), 289-297.

[3] L. Carlitz and Jack Levine, An identity of Cayley, American Mathematical Monthly 67 (1960), 571-573.

[4] A. Cayley, Note sur les normales d'une conique, Crelle's Journal 56 (1859), 182-185.

[5] A. G. Izergin, Partition function of a six-vertex model in a finite volume (Russian), Dokl. Akad. Nauk SSSR 297 (1987), 331-333.

[6] V. E. Korepin, N. M. Bogoliubov, and A. G. Izergin, Quantum Inverse Scattering Method and Correlation Functions, Cambridge: Cambridge University Press, 1993.

[7] G. Kuperberg, Another proof of the alternating sign matrix conjecture, International Mathematics Research Notes (1996), 139-150.

[8] T. Muir, A Treatise on Determinants, London, 1881.

[9] D. Zeilberger, Proof of the refined alternating sign matrix conjecture, New York Journal of Mathematics 2 (1996), 59-68. 